\title{
Article \\ Metabolomic Investigation of Synergistic Mechanism for Fangfeng Extract Preventing LPS Induced Neuroinflammation in BV-2 Microglia Cells
}

\author{
Xinliang Zhu ${ }^{1,2,3,4, *}$, Xueqi Wang ${ }^{1}$, Shunbin Zhang ${ }^{1}, \mathrm{Xu} \mathrm{Li}^{1}{ }^{1}$, Zhengdou Li ${ }^{1}$, Xia Chen ${ }^{1}$, Xiaoxiao Liu ${ }^{5}$, \\ Huixia $\mathrm{Li}^{6}$ and Ji Zhang ${ }^{1,2,3, *}$ \\ 1 College of Life Science, Northwest Normal University, Lanzhou 730070, China; \\ 2020119145@nwnu.edu.cn (X.W.); 2019212301@nwnu.edu.cn (S.Z.); 201674010414@nwnu.edu.cn (X.L.); \\ 2020212640@nwnu.edu.cn (Z.L.); 2020212643@nwnu.edu.cn (X.C.) \\ 2 Bioactive Products Engineering Research Center for Gansu Distinctive Plants, Lanzhou 730070, China \\ 3 Institute of Rural Development and Research, Northwest Normal University, Lanzhou 730070, China \\ 4 School of Life Science and Technology, Xi'an Jiaotong University, Xi'an 710049, China \\ 5 Lanzhou Institute of Food and Drug Control, Lanzhou 740050, China; 1xx0808@163.com \\ 6 Gansu Tongxing Intelligent Technology Development Co., Ltd., Lanzhou 730070, China; lhx0520@163.com \\ * Correspondence: xzhuaf@nwnu.edu.cn (X.Z.); zhangj@nwnu.edu.cn (J.Z.)
}

\section{check for}

updates

Citation: Zhu, X.; Wang, X.; Zhang, S.; Li, X.; Li, Z.; Chen, X.; Liu, X.; Li, H.; Zhang, J. Metabolomic Investigation of Synergistic Mechanism for Fangfeng Extract Preventing LPS Induced Neuroinflammation in BV-2 Microglia Cells. Appl. Sci. 2021, 11, 8155. https://doi.org/10.3390/ app11178155

Academic Editor: Marcello Iriti

Received: 13 July 2021

Accepted: 27 August 2021

Published: 2 September 2021

Publisher's Note: MDPI stays neutral with regard to jurisdictional claims in published maps and institutional affiliations.

Copyright: (C) 2021 by the authors Licensee MDPI, Basel, Switzerland. This article is an open access article distributed under the terms and conditions of the Creative Commons Attribution (CC BY) license (https:// creativecommons.org/licenses/by/ $4.0 /)$.
Abstract: The root of Saposhnikovia divaricata (Fangfeng) is commonly used in traditional Chinese medicine (TCM) for headache and neuroinflammation-related disease treatment. The mRNA expression of IL- 6 and IL-1 $\beta$ were significantly inhibited after Fangfeng extract (FFE) treatment in LPS-induced BV-2 cells. Metatolome profiling indicated that dopamine, palmitic acid, corticosterone, and eicosapentaenoic acid metabolites could be regulated by FFE for LPS stimulated inflammation responses in BV-2 cells. The disturbed metabolic pathways include caffeine metabolism, mannose type O-glycan biosynthesis, arachidonic acid metabolism, and steroid biosynthesis. This study will enable us to identify potential protein targets and metabolite intermediates for FFE exerting its protective function in BV-2 cells, and it also provided a potential application of Fangfeng in neuroinflammation-related disease treatment.

Keywords: Saposhnikovia divaricata; neuroinflammation; BV-2 cells; metabolomics; cytokine

\section{Introduction}

Many neuronal diseases might be activated by inflammatory processes and their specific mediators [1]. Patients with neurodegenerative diseases have been recognized for extensive pro-inflammatory cytokines expression in the brains, cerebrospinal fluid, and serum without infiltration of adaptive immune cells into the CNS (Central Nerve System) [2]. The fibrillar A $\beta$ (amyloid- $\beta$ ) could induce microglial activation and cytokines secretion with AD compared with healthy controls [3]. $\alpha$-synucein might activate inflammatory responses and induce IL-1 $\beta$ release and NLRP3 expression in a PD animal model [4]. The mutant huntingtin resulted in hyperinflammatory microglia and activated the key myeloid transcription factors Pu.1 and C/EBP (CCAAT/Enhancer Binding Protein) in microglia [5]. In this perspective, neuroinflammation cascade could be crucial for neurodegenerative diseases. According to ancient Chinese medicinal monographs, Shennong Bencao Jing, Fangfeng (the root of Saposhnikovia divaricata, SD) could relieve spasms through expelling endogenous wind and dispelling pathogenic wind to [6-8]. Fangfeng is extensively applied in traditional Chinese medicine (TCM) for treating headaches, joint pain, and rheumatic arthritis [9]. Pharmacological studies have shown that Fangfeng has neuroprotective effects [10-12]. It recently has been indicated to have neuroprotective effects through inhibiting $\mathrm{H}_{2} \mathrm{O}_{2}$-induced toxicity and $\mathrm{A} \beta$-induced cell death and acetylcholinesterase (AChE) activity in preliminary experiments [13]. Other studies revealed 
Fangfeng could improve focal cerebral ischemia-induced neuronal damage using novel Guhpoongchungsimhwan (GCH) herbal decoctions and decrease abnormal amyloid precursor protein accumulation and protect neurovascular internal units using Houshiheisan compound prescription [14,15]. Moreover, one of the active ingredients of Fangfeng, the 4'-O- $\beta$-d-glucosyl-5-O-methylvisamminol (4OGOMV), exhibits neuroprotective function through restraining IL-6 (interleukin-6) and MCP-1 stimulation and HMGB1 expression, while also decreasing the cytokine mRNA and inflammasome formation in subarachnoid hemorrhage (SAH)-induced vasospasm [16]. As described above, through traditional Chinese medicine and biological experiments, we speculate that Fangfeng may exert neuroprotective functions through attenuating neuroinflammation processes. Microglia are the main effector cells of neuroinflammatory response, which can produce TNF- $\alpha$, IL-1 $\beta$ after over activation [17]. Therefore, inhibition of the microglia-mediated neuroinflammatory response may be a potential treatment for neurodegenerative diseases [18]. Metabolomics, an emerging analytical methodology in systems biology, was applied to investigate the metabolic changes of endogenous metabolites in living systems to pathological stimuli or drug treatments. By high-throughput detection and data processing of endogenous metabolites present in biological fluids or tissues, metabolomics could find potential biomarkers related to physiological or pathological changes [19,20]. Liquid Chromatography-Mass Spectrometry (LC-MS) is one of the most commonly used analytical techniques in metabolomics. The holistic and systematic characteristics of metabolomics make it suitable for the analysis of complex systems such as TCM, which exerts its efficacy through multi-components and multi-targets. At present, metabolomics has been widely applied to study the pharmacological mechanism of TCM, including the mechanism of TCM in treating neurodegenerative diseases [21]. In this study, the LPS-stimulated inflammation model of BV-2 cells was established, and the metabolome spectrum of BV-2 cells was profiled by a mass spectrometry-based metabolomics approach with or without FFE protection. We attempted to conduct an untargeted metabolomics investigation to disclose the potential and molecular mechanism of FFE for neuroinflammation protection.

\section{Materials and Methods}

\subsection{Materials}

The roots of Saposhnikovia divaricata (Fangfeng) were purchased from Lanzhou Focixicheng Pharmaceutical Co., Ltd. (Lanzhou, Gansu, China) and were authenticated by Prof. Fude Yang (Gansu University of Traditional Chinese Medicine, Lanzhou, China). Lipopolysaccharide (LPS). High-glucose Dulbecco's modified Eagle's medium (DMEM) and fetal bovine serum (FBS) were purchased from Sigma-Aldrich (St. Louis, MO, USA). The $0.05 \%$ trypsin and penicillin-streptomycin (PS) were both obtained from Invitrogen (Carlsbad, CA, USA). The FastKing gDNA Dispelling RT SuperMix kit and the SYBR Green QuantiTect RT-PCR Kit were purchased from TIANGEN (Beijing, China).

Preparation of the root of Saposhnikovia divaricata (Fangfeng) water extract: after grinding, we weighed $50 \mathrm{~g}$ of Fangfeng, and immersed it in $400 \mathrm{~mL} \mathrm{30 \%} \mathrm{ethanol} \mathrm{in} \mathrm{distilled}$ water and extracted under reflux at $80^{\circ} \mathrm{C}$ for $4 \mathrm{~h}$. After filtering, it was centrifuged for $10 \mathrm{~min}$ at $4000 \mathrm{rpm}$, the supernatant was collected, and freezer dried and stored in a $-80^{\circ} \mathrm{C}$ freezer for further use.

\subsection{Cell Culture and Drug Treatment}

Murine microglia BV-2 cells were purchased from Hongshun Biologicals (Shanghai, China) and maintained in high-glucose Dulbecco's modified Eagle's medium (DMEM) supplemented with $10 \%$ fetal bovine serum (FBS), $100 \mathrm{U} / \mathrm{mL}$ penicillin (Beyotime, Shanghai, China) and $100 \mu \mathrm{g} / \mathrm{mL}$ streptomycin (Beyotime, Shanghai, China) at $37^{\circ} \mathrm{C}$, in a $5 \% \mathrm{CO}_{2}$ humidified incubator. The cell and animal experiment protocol was approved by the animal ethics committee of Xi'an Jiaotong University's School of Life Science and Technology (approval Nr. SCXK (Shaan) 2017-003). FFE was freshly dissolved in the cell culture medium. Either FFE-containing medium or regular medium was applied for inflammation model 
BV-2 cells, which were built by $12 \mathrm{~h}$ of $1 \mathrm{mM}$ of LPS pretreatment. Cells were treated with FFE-containing medium for $12 \mathrm{~h}$ with different concentrations $(0.1 \mathrm{mg} / \mathrm{mL}, 1 \mathrm{mg} / \mathrm{mL}$ and $10 \mathrm{mg} / \mathrm{mL}$ refers to QF 0.1 , QF 1 and QF 10 groups, respectively) before RNA or metabolite extraction, as indicated.

\subsection{Real-Time PCR Assay}

The total RNA of BV-2 cells were obtained by using a Trizol reagent according to the manufacturer's instructions. After measurement of RNA concentration using an absorbance ratio of the spectrophotometer at $260 \mathrm{~nm}$ and $280 \mathrm{~nm}$, we reverse-transcribed (RT) RNA into cDNA using the FastKing gDNA Dispelling RT SuperMix kit according to the manufacturer's instructions. The primer sequences were as follows: $\beta$-actin: $5^{\prime}$-GTCAGGTCATCACTATCGGCAAT-3' (forward) and 5'-AGAGGTCTTTACGGATGTCA ACGT-3' (reverse); IL-6: 5' -GCCCACCAAGAACGATAGTCA-3' (forward) and 5'-ACCAGC ATCAGTCCCAAGAAG- $3^{\prime}$ (reverse); IL-1 $\beta$ : $5^{\prime}$-TCACAAGCAGAGCACAAGCC-3' (forward) and $5^{\prime}$-CATTAGAAACAGTCCAGCCCATAC-3' (reverse). The real-time PCR was then analyzed with a SYBR Green QuantiTect RT-PCR Kit, and each sample was independently analyzed three times. The PCR amplification was performed with 40 cycles of denaturation at $95^{\circ} \mathrm{C}$ for $10 \mathrm{~s}$, annealing at $55^{\circ} \mathrm{C}$ for $20 \mathrm{~s}$ and extension at $72{ }^{\circ} \mathrm{C}$ for $30 \mathrm{~s}$. The relative levels of gene expression of each mRNA were calculated by normalization to $\beta$-actin mRNA expression according to the $2^{-\Delta \Delta C T}$ method.

\subsection{Statistical Analysis}

The data statistical significance was evaluated using single-factor analysis of variance (one-way ANOVA) and Tukey's range test via the PrismDemo software (GraphPad Softwar, San Diego, CA, USA). The results were considered significant when the $p$-value was $\leq 0.05$.

\subsection{Sample Preparation for $L C-M S / M S$}

Cells were collected after FFE treatment and washed with cold phosphate buffer (PBS) with $\mathrm{pH}$ value of 7.43 times, scraped from the ice plate with a plastic cell scraper, and collected in a $1.5 \mathrm{~mL}$ tube. One $\mathrm{mL}$ of extraction buffer (acetonitrile:methanol:water $=2: 2: 1$ ) was added to the collected BV-2 cells. A quality control (QC) sample was prepared by mixing of $10 \mu \mathrm{L}$ of each test sample. Samples were freezer dried and stored at $-80{ }^{\circ} \mathrm{C}$ before LC-MS analysis.

\subsection{LC-MS Analysis}

Acetonitrile aqueous solution (acetonitrile:water $=2: 1,400 \mu \mathrm{L}$ ) was added into the samples respectively. Then, the mixture was vortexed for $1 \mathrm{~min}$ and centrifuged at $14,000 \times g$ for $15 \mathrm{~min}$ at $4{ }^{\circ} \mathrm{C}$. Finally, the obtained supernatant was transferred to vials for analysis. Chromatography separation was carried out on a Thermo Hypersil Gold C18 column $(100 \mathrm{~mm} \times 2.1 \mathrm{~mm}, 1.8 \mu \mathrm{m})$ at $40{ }^{\circ} \mathrm{C}$. Mobile phase A: $0.1 \%$ formic acid aqueous solution $(v / v)+$ acetonitrile, phase B: $5 \mathrm{mM}$ ammonium acetate solution + acetonitrile. The gradient elution program: 0 min-2 min, 1-15\% B; 2 min-5 min, 15-55\% B; 5 min-8 min, 55-99\% B; $10 \mathrm{~min}-12 \mathrm{~min}, 99-1 \% \mathrm{~B}$. The flow rate was $0.5 \mathrm{~mL} / \mathrm{min}$. Column temperature was set as $35{ }^{\circ} \mathrm{C}$ and the sample injection volume was $3 \mu \mathrm{L}$. Mass spectrometry data were collected both in positive and negative ion modes of the ESI source of Q Exactive Orbitrap Mass Spectrometer. Using Full MS (Resolution 70000) and dd-MS2/dd-SIM (Resolution $17500)$, the scanning range was $100-1500 \mathrm{~m} / \mathrm{z}$, the capillary temperature was 400 degrees, and the auxiliary gas heater temperature was 350 degrees Celsius. The analytical method for the QC samples was the same as other samples and was injected after each of 6 realsample injections.

\subsection{Metabolomics Data Analysis}

The metabolite profiling data were obtained and uploaded to the cloud-based XCMS online platform for data processing and peak detection, retention time correction, profile 
alignment, and the peak area information were standardized in Microsoft Excel 2010. Then, principal component analysis (PCA), partial least squares discriminant analysis (PLS-DA), orthogonal partial least squares discriminant analysis (OPLS-DA), and volcanic map were used to further analyze the standardized data in metabolic analysis 5.0 online server (http:/ / www.metaboanalyst.ca (accessed on 23 April 2021)). Finally, the differential metabolites were obtained based on variable importance in projection (VIP) value (VIP $>1$ ) and $t$ test $(p<0.05)$. The metabolic pathways of significantly differential accumulated biomarkers were analyzed by MetaboAnalyst 5.0 and Kyoto Encyclopedia of Genes and Genomes (KEGG) pathways.

\section{Results}

\subsection{Protective Effect of Fangfeng Extract}

To confirm the protective effects of Fangfeng extract (FFE) on inflammatory response in LPS-stimulated BV-2 cells, we firstly confirmed the prominent changes of pro-inflammatory cytokine production. The mRNA levels of pro-inflammatory cytokines (IL- 6 and IL-1 $\beta$ ) were measured by real-time PCR. As shown in the figure, the relative expression of IL- 6 and IL-1 $\beta$ were significantly increased after LPS induction in BV-2 cells $(p<0.01)$. Meanwhile, results indicated that FFE observably inhibited gene expression levels of pro-inflammatory cytokines (IL-6 and IL-1 $\beta$ ) in a dose-dependent manner (Figure 1).
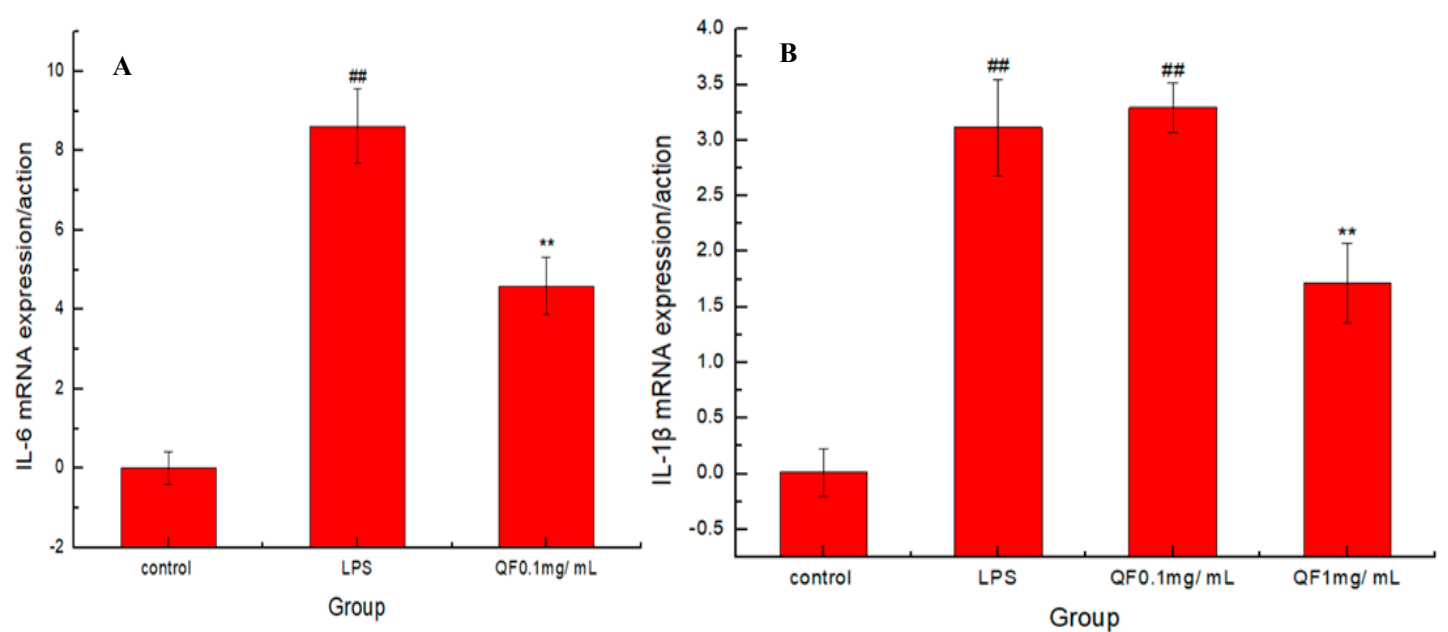

Figure 1. Effects of Fangfeng extract on the production of pro-inflammatory cytokines (IL-6 and IL-1 $\beta$ ) in LPS-induced BV-2 cells. (A) Effect on the production of proinflammatory cytokines IL-6, (B) Effect on the production of proinflammatory cytokines IL-1 $\beta$. All the experiments were repeated at least three times and similar results were observed. Values are mean $\pm \mathrm{SE}$, $\left(n=4\right.$ samples/group). ${ }^{\# \#} p<0.01$ vs. control group, ${ }^{* *} p<0.01$ vs. LPS-treated group.

\subsection{Metabolomics Profiling and Differential Accumulated Metabolite (DAM) Analysis}

OPLS-DA analysis showed that the control group and LPS treatment group were far away from FFE-treated groups, meaning FFE and LPS could greatly disturb the metabolic profile of BV-2 cells. Drug-treated groups were effectively separated between different concentrations (Figure 2).

In order to identify DAM, VIP value $(>1.0)$, fold change (FC $\geq 2$ or $\mathrm{FC} \leq 0.5)$ and $t$-test $p$ value $(p<0.05)$ in volcano map (Figure 3$)$ and OPLS-DA analysis were used as selection criteria. The DAM details can be found in supplementary file S1, such as levodopadopamine, docosahexaenoic acid, gamma hydroxybutyric acid, 5-hydroxy-Ltryptophan, alpha-tocopherol, etc., which were upregulated by FFE pre-protection when compared with LPS treatment. MetaboAnalyst software was used for metabolomic pathway enrichment analysis. In the negative mode (Table 1), for the LPS group versus QF 0.1 group, alanine, aspartate and glutamate metabolism, purine metabolism, and pyrimidine metabolism were the top upregulated pathways (Figure 4A); for the LPS group 
versus QF 1 group, purine metabolism, alanine, aspartate, and glutamate metabolism and aminoacyl-tRNA biosynthesis were the main upregulated pathways (Figure 4B); for the LPS group versus the QF 10 group, tyrosine metabolism, butanoate metabolism, and galactose metabolism were the main upregulated pathways (Figure $4 \mathrm{C}$ ). In the positive mode, for the LPS group versus QF0.1 group, butanoate metabolism, arginine biosynthesis and starch, and sucrose metabolism were major upregulated pathways (Figure 4D); for the LPS group versus the QF1 group, primary bile acid biosynthesis, taurine and hypotaurine metabolism, and tryptophan metabolism were the most enriched upregulated pathways (Figure 4E); for the LPS group versus the QF10 group, tryptophan metabolism, sphingolipid metabolism, and tyrosine metabolism were the top enriched upregulated pathways (Figure 4F).
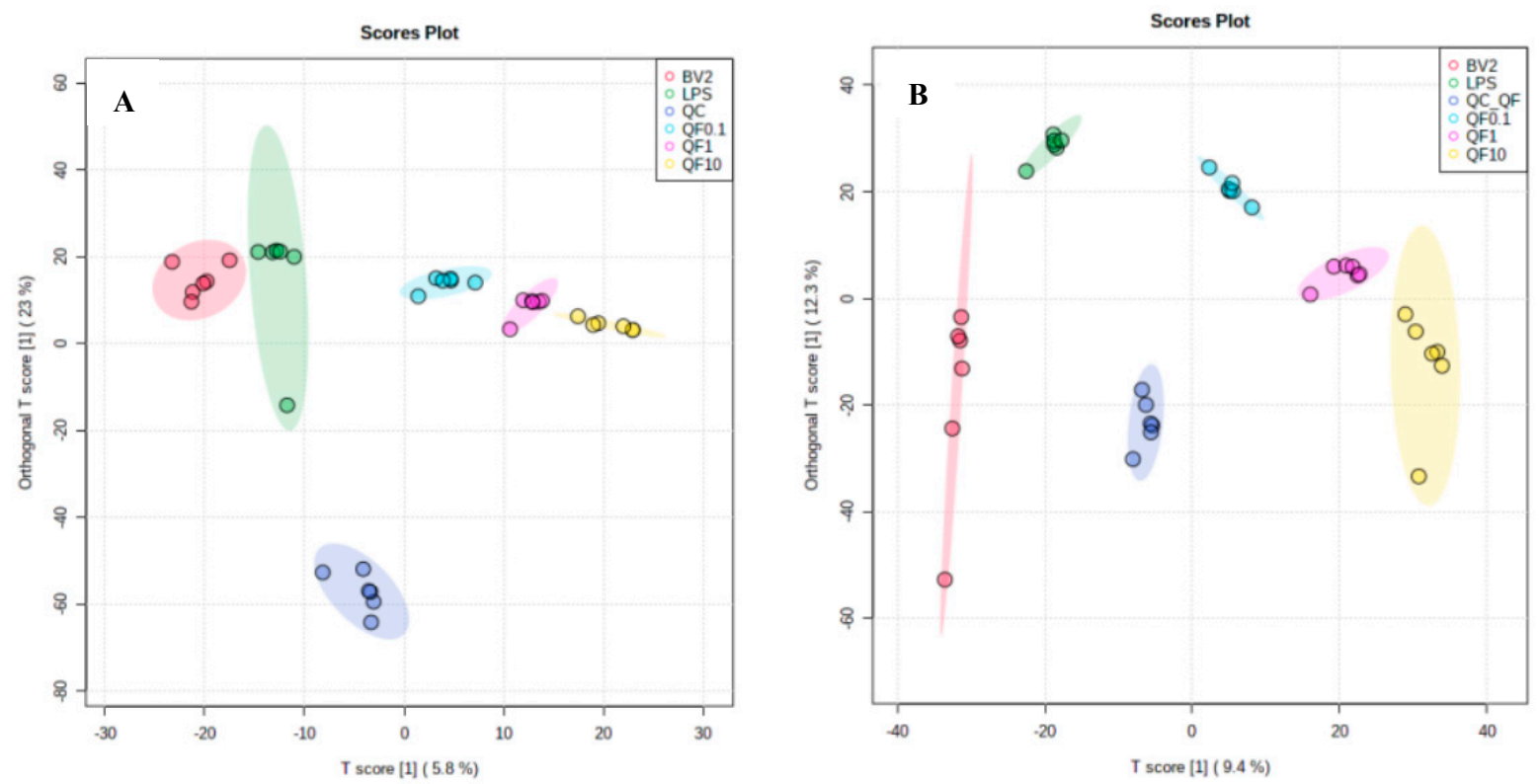

Figure 2. Negative OPLS-DA score map (A) and positive OPLS-DA score map (B).

Table 1. Enrichment maps' description of upregulated metabolic pathways.

\begin{tabular}{|c|c|}
\hline Figures & Data Points (Metabolic Pathways) \\
\hline (A) LPS vs. QF0.1 (negative) & $\begin{array}{l}\text { 1. Alanine, aspartate and glutamate metabolism; } 2 \text {. Purine metabolism; } 3 \text {. Pyrimidine metabolism; } 4 \text {. Steroid } \\
\text { biosynthesis; } 5 \text {. Starch and sucrose metabolism; } 6 \text {. Butanoate metabolism }\end{array}$ \\
\hline (B) LPS vs. QF1 (negative) & $\begin{array}{l}\text { 1. Purine metabolism; 2. Alanine, aspartate, and glutamate metabolism; } 3 \text {. Aminoacyl-tRNA biosynthesis; } \\
\text { 4. Butanoate metabolism; } 5 \text {. Selenocompound metabolism; } 6 \text {. Citrate cycle (TCA cycle); } 7 \text {. Tyrosine metabolism } \\
\text { 1. Tyrosine metabolism; } 2 \text {. Butanoate metabolism; } 3 \text {. Galactose metabolism; } 4 \text {. Porphyrin and chlorophyll }\end{array}$ \\
\hline (C) LPS vs. QF10 (negative) & $\begin{array}{l}\text { metabolism; 5. Taurine and hypotaurine metabolism; 6. Citrate cycle (TCA cycle); 7. Purine metabolism; } \\
\text { 8. Propanoate metabolism; } 9 \text {. Alanine, aspartate, and glutamate metabolism }\end{array}$ \\
\hline (D) LPS vs. QF0.1 (positive) & $\begin{array}{l}\text { 1. Butanoate metabolism; } 2 \text {. Arginine biosynthesis; } 3 \text {. Starch and sucrose metabolism } 4 \text {. Sphingolipid } \\
\text { metabolism; } 5 \text {. D-Glutamine and D-glutamate metabolism; } 6 \text {. Tyrosine metabolism; } 7 \text {. Purine metabolism; } \\
\text { 8. Primary bile acid biosynthesis }\end{array}$ \\
\hline (E) LPS vs. QF1 (positive) & $\begin{array}{l}\text { 1. Primary bile acid biosynthesis; } 2 \text {.Taurine and hypotaurine metabolism; } 3 \text {. Tryptophan metabolism; } 4 \text {. Retinol } \\
\text { metabolism }\end{array}$ \\
\hline (F) LPS vs. QF10 (positive) & $\begin{array}{l}\text { 1. Tryptophan metabolism; } 2 \text {. Sphingolipid metabolism; } 3 \text {. Tyrosine metabolism; } 4 \text {. Retinol metabolism; } \\
\text { 5. Histidine metabolism; } 6 \text {. Pentose and glucuronate interconversions; } 7 \text {. Pentose phosphate pathway } \\
\text { 1. Primary bile acid biosynthesis; } 2 \text {. One carbon pool by folate; } 3 \text {. Nicotinate and nicotinamide metabolism; }\end{array}$ \\
\hline (G) control vs. LPS (positive) & $\begin{array}{l}\text { 1. Primary bile acid biosynthesis; } 2 \text {. One carbon pool by folate; } 3 \text {. Nicotinate and nicotinamide metabolism; } \\
\text { 4. Vitamin B6 metabolism; } 5 \text {. Tryptophan metabolism; } 6 \text {. beta-Alanine metabolism; } 7 \text {. Pentose phosphate } \\
\text { pathwav; } 8 \text {. Pyruvate metabolism }\end{array}$ \\
\hline (H) control vs. LPS (negative) & 1.Vitamin B6 metabolism; 2 . Primary bile acid biosynthesis; 3 . Fatty acid biosynthesis \\
\hline
\end{tabular}



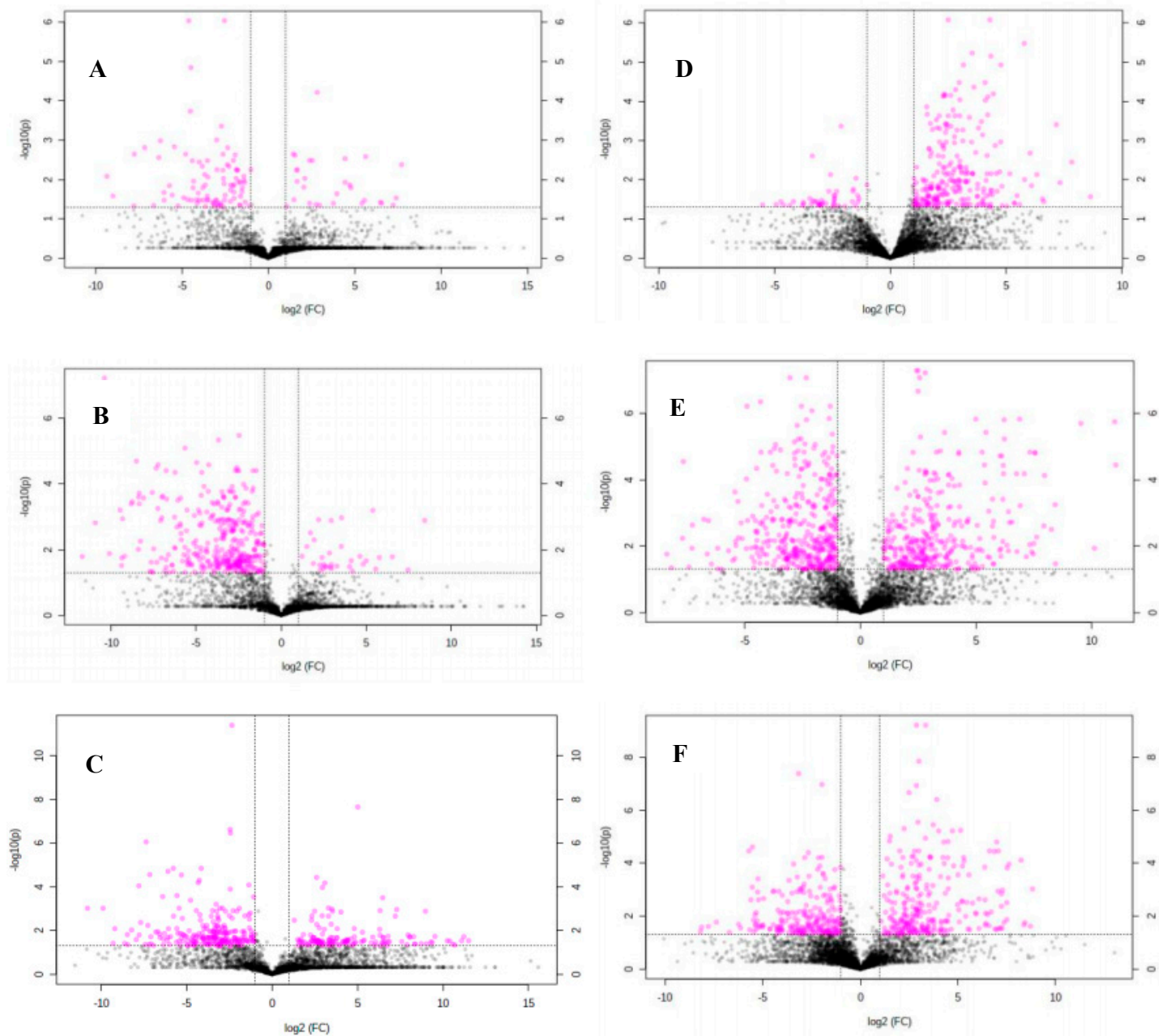

Figure 3. Metabolite volcano map in negative ion mode: (A) LPS vs. QF 0.1; (B) LPS vs. QF 1; (C) LPS vs. QF 10; metabolite volcano map in positive ion mode: (D) LPS vs. QF 0.1; (E) LPS vs. QF 1; (F) LPS vs. QF 10.
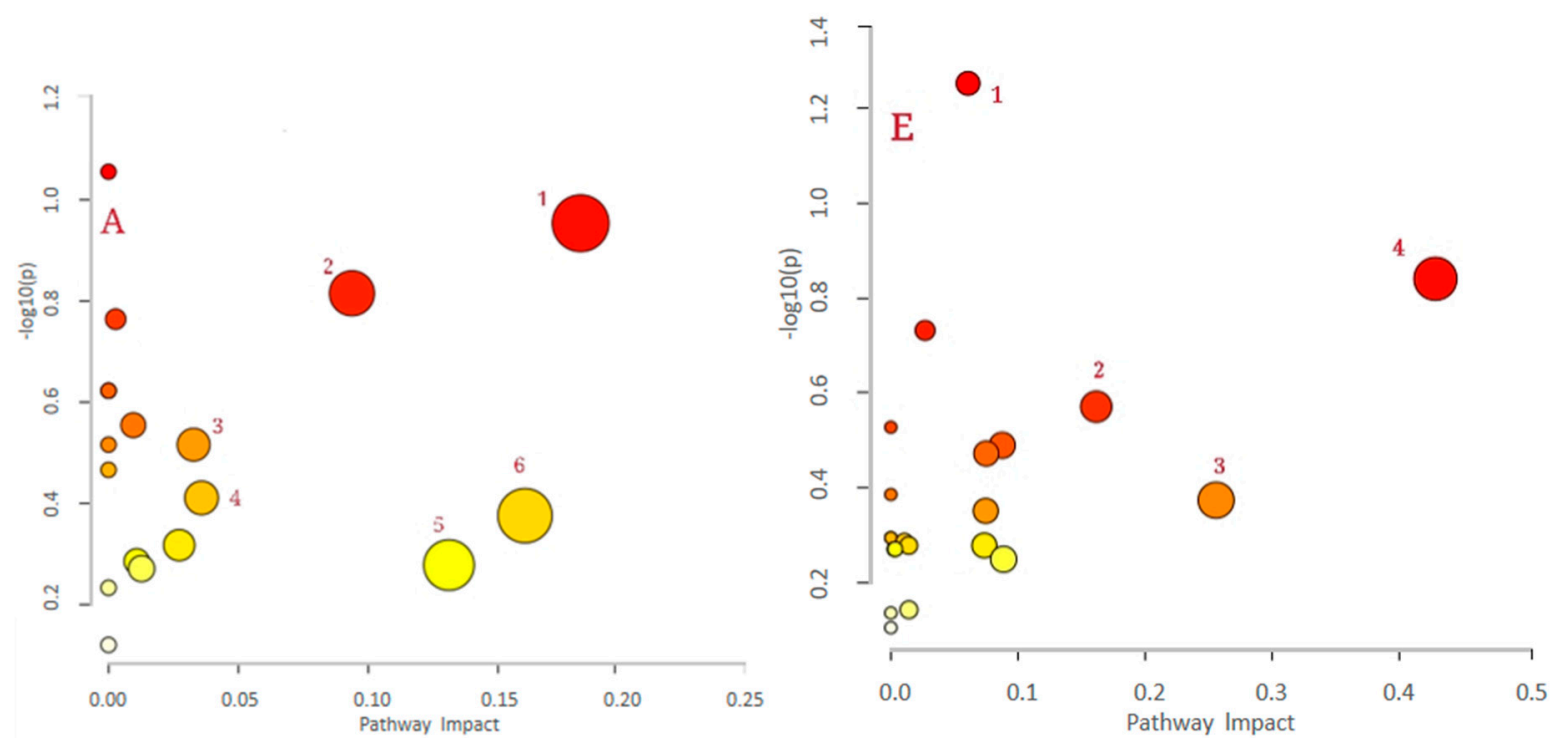

Figure 4. Cont. 

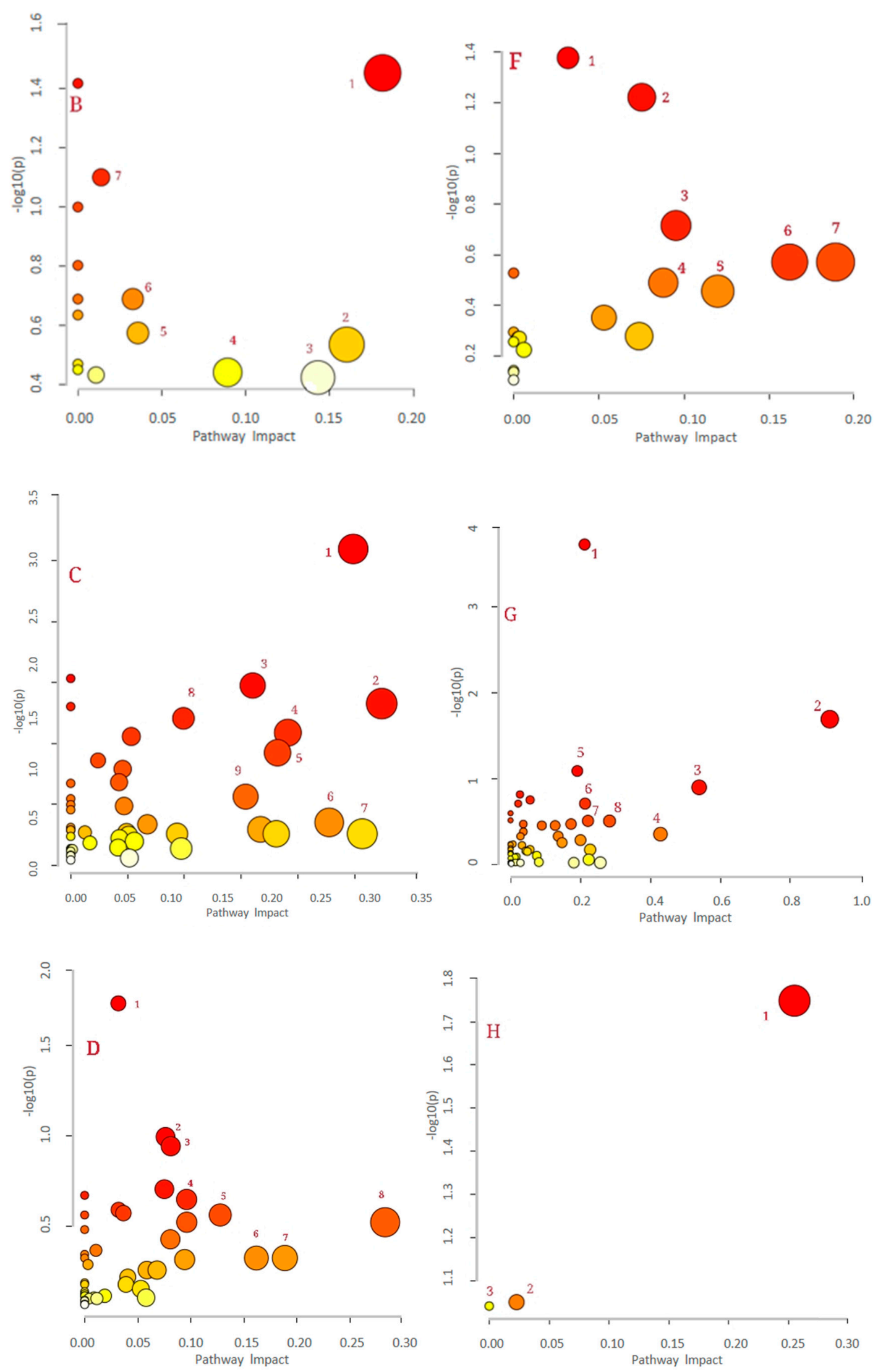

Figure 4. Enrichment maps of upregulated metabolic pathways in negative and positive modes. Negative ion mode: (A) LPS vs. QF 0.1; (B) LPS vs. QF 1; (C) LPS vs. QF 10; (H) Control vs. LPS.Positive ion mode: (D) LPS vs. QF 0.1; (E) LPS vs. QF 1; (F) LPS vs. QF 10; (G) Control vs. LPS. Data point descriptions are in Table 1. 
Additionally, the downregulated DAM were also included in Supplementary File S1, such as folate, arachidonic acid, Prostaglandins, 12(S)-HPETE, 3-ketosphinganine, Galactosylsphingosine etc., and were downregulated by FFE pre-protection when compared with LPS treatment. Metabolomic pathway enrichment analysis were also performed and the results were as follows: in the negative mode (Table 2), for the LPS group versus the QF 0.1 group, thiamine metabolism, ether lipid metabolism, and galactose metabolism were the top downregulated pathways (Figure 5A); for the LPS group versus the QF 1 group, one carbon pool by folate, biosynthesis of unsaturated fatty acids, and Purine metabolism were the main downregulated pathways (Figure 5B); for the LPS group versus the QF 10 group, one carbon pool by folate, porphyrin and chlorophyll metabolism, and thiamine metabolism were the main upregulated pathways (Figure 5C). In the positive mode, for the LPS group versus the QF0.1 group, steroid biosynthesis, one carbon pool by folate, and nicotinate and nicotinamide metabolism were the major downregulated pathways (Figure 5D); for the LPS group versus the QF1 group, one carbon pool by folate, sphingolipid metabolism, and sulfur metabolism were the most enriched downregulated pathways (Figure 5E); for the LPS group versus the QF10 group, one carbon pool by folate, glycine serine, and threonine metabolism and sulfur metabolism were the top enriched downregulated pathways (Figure 5F).

The metabolic pathways enrichment analysis results of all groups are listed in Table 2.

Table 2. Enrichment maps' description of downregulated metabolic pathways.

\begin{tabular}{|c|c|}
\hline Figures & Data Points (Metabolic Pathways) \\
\hline (A) LPS vs. QF0.1 (negative) & $\begin{array}{l}\text { 1. Sphingolipid metabolism; } 2 \text {. One carbon pool by folate; } 3 \text {. Phenylalanine metabolism; } 4 \text {. Tyrosine } \\
\text { metabolism; } 5 \text {. Nicotinate and nicotinamide metabolism; } 6 \text {. Histidine metabolism }\end{array}$ \\
\hline (B) LPS vs. QF1 (negative) & $\begin{array}{l}\text { 1. Steroid biosynthesis; } 2 \text {. One carbon pool by folate; } 3 \text {. Nicotinate and nicotinamide metabolism; } \\
\text { 4. Mannose type O-glycan biosynthesis }\end{array}$ \\
\hline (C) LPS vs. QF10 (negative) & $\begin{array}{l}\text { 1. One carbon pool by folate; } 2 \text {. Sphingolipid metabolism; } 3 \text {. Sulfur metabolism; } 4 \text {. Steroid biosynthesis; } \\
\text { 5. Biotin metabolism; } 6 \text {. Primary bile acid biosynthesis }\end{array}$ \\
\hline (D) LPS vs. QF0.1 (positive) & $\begin{array}{l}\text { 1. One carbon pool by folate; } 2 \text {. Glycine serine and threonine metabolism; } 3 \text {. Sulfur metabolism; } \\
\text { 4. Phenylalanine metabolism; } 5 \text {. Nicotinate and nicotinamide metabolism; } 6 \text {. Fructose and mannose } \\
\text { metabolism; } 7 \text {. Sphingolipid metabolism }\end{array}$ \\
\hline (E) LPS vs. QF1 (positive) & $\begin{array}{l}\text { 1. Primary bile acid biosynthesis; } 2 \text {. Pyrimidine metabolism; } 3 \text {. Tyrosine metabolism; } 4 \text {. Steroid } \\
\text { hormone biosynthesis; } 5 \text {. Synthesis and degradation of ketone bodies }\end{array}$ \\
\hline (F) LPS vs. QF10 (positive) & $\begin{array}{l}\text { 1. Thiamine metabolism; 2. Ether lipid metabolism; } 3 \text {. Galactose metabolism; } 4 \text {. Porphyrin and } \\
\text { chlorophyll metabolism }\end{array}$ \\
\hline (G) control vs. LPS (positive) & $\begin{array}{l}\text { 1. One carbon pool by folate; } 2 \text {. Biosynthesis of unsaturated fatty acids; } 3 \text {. Purine metabolism; } 4 \text {. Fatty } \\
\text { acid biosynthesis; } 5 \text {. Synthesis and degradation of ketone bodies; } 6 \text {. Porphyrin and chlorophyll } \\
\text { metabolism; } 7 \text {. Thiamine metabolism; } 8 \text {. Taurine and hypotaurine metabolism; } 9 \text {. Sulfur metabolism; } \\
\text { 10. Glycerophospholipid metabolism }\end{array}$ \\
\hline (H) control vs. LPS (negative) & $\begin{array}{l}\text { 1. One carbon pool by folate; } 2 \text {. Porphyrin and chlorophyll metabolism; } 3 \text {. Thiamine metabolism; } \\
\text { 4. Pyrimidine metabolism; } 5 \text {. Starch and sucrose metabolism; } 6 \text {. Pantothenate and CoA biosynthesis; } \\
\text { 7. Ether lipid metabolism; } 8 \text {. Sphingolipid metabolism; } 9 \text {. beta-Alanine metabolism }\end{array}$ \\
\hline
\end{tabular}



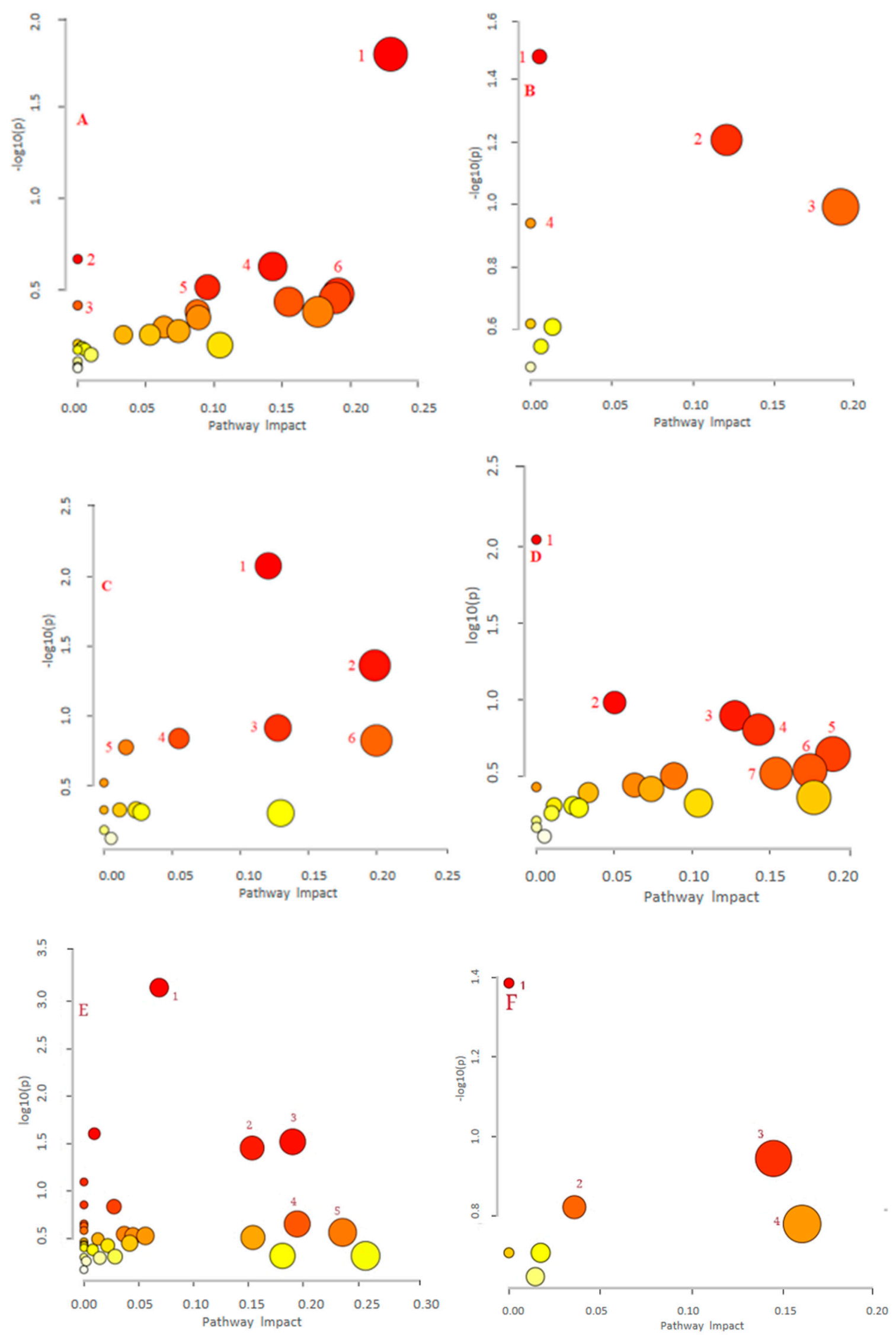

Figure 5. Cont. 

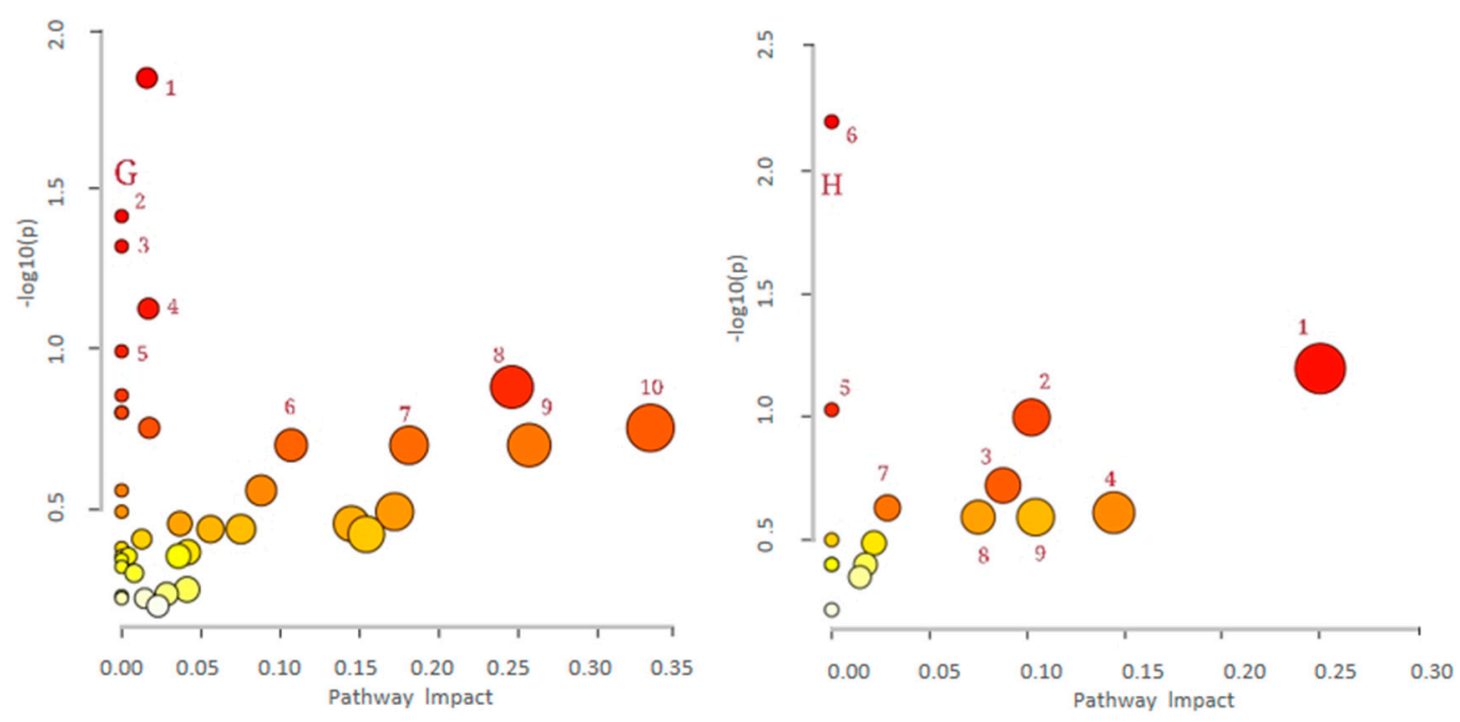

Figure 5. Enrichment maps of downregulated metabolic pathways in negative and positive modes. Negative ion mode: (A) LPS vs. QF 0.1; (B) LPS vs. QF 1; (C) LPS vs. QF 10; (H) Control vs. LPS. Positive ion mode: (D) LPS vs. QF 0.1; (E) LPS vs. QF 1; (F) LPS vs. QF 10; (G) Control vs. LPS. Data point descriptions are in Table 2.

\section{Discussion}

Neuroinflammation is widely observed in neurodegenerative diseases, which might have been affected by glia cells activation and cytokines secretion. Fangfeng may play a neuroprotective function by reducing the process of neuroinflammation, though the detailed mechanisms are not clear.

In our study, metabolite-profiling uncovered levodopa, docosahexaenoic acid, gamma hydroxybutyric acid, 5-hydroxy-L-tryptophan, alpha-tocopherol, etc., metabolites were upregulated by FFE in LPS-induced BV-2 cells. Levodopa is the precursor of dopamine, while dopamine is a neurotransmitter which has been shown to have anti-inflammatory effects $[22,23]$. In the peripheral nervous system, dopamine receptor activation can control tissue inflammation and injury [24]. In our study, docosahexaenoic acid and gamma hydroxybutyric acid were also significantly upregulated upon FFE treatment compared with the LPS group. Research has shown that docosahexaenoic acid inhibits neuroinflammation via its conversion to specialized pro-resolving mediators and decreased oxidative stress and microglial pro-inflammatory activation $[25,26]$, while gamma hydroxybutyric acid, which is derived from $\gamma$-aminobutyric acid (GABA) [27], may act a neuromodulator in the CNS and has several neuronal mechanisms such as activation of the GABA receptor. Multiple studies revealed that alpha-tocopherol has an important role in antioxidant protection of neurodegenerative diseases and dietary tocopherol intake could improve PD-related parameters [28]. The results of metabolic pathway enrichment analysis indicated that the main upregulated metabolic pathways were alanine, aspartate and glutamate metabolism, purine metabolism, tyrosine metabolism, butanoate metabolism, primary bile acid biosynthesis, taurine and hypotaurine metabolism, tryptophan metabolism, and sphingolipid metabolism, etc. These results indicate that the differential metabolites of BV2 cells treated with FFE can effectively inhibit cell inflammation through amino acid and fatty acid metabolism and neurotransmitter and neuro-protective metabolites derived from them. The tyrosine and tryptophan metabolisms were demonstrated as crucial sources of neurotransmitters tightly associated with neuropsychiatric symptoms [29]. Besides, multiple amino acid-derived intermediates were critical for the Alzheimer's disease pathological progression through immune cells infiltration-derived neuroinflammation [30]. At the same time, metabolites like folate, arachidonic acid, Prostaglandins, 12(S)-HPETE, 3-ketosphinganine, Galactosylsphingosine, and etc. were downregulated by FFE preprotection when compared with LPS treatment. The downregulated pathways include: one carbon pool by folate, biosynthesis of unsaturated fatty acids, sphingolipid metabolism, 
and steroid biosynthesis. The impaired one-carbon metabolism and lower folate levels tightly correlated with elevated tau phosphorylation levels in neurodegenerative diseases and disturbed neuronal homeostasis [31]. However, recent research on the genetic and supplementation-manipulated $C$. elegans indicated that specific folate and one carbon metabolism reduction could be beneficial for longevity and proteoprotection [32]. These disparate observations probably reflect multi-layering of FFE effects on LPS-induced BV-2 cells since it could effectively decrease pro-inflammatory factor expression and downregulate folate metabolisms. Additionally, unsaturated fatty acids, sphingolipid, and steroid metabolism alteration in FFE-protected microglia cells reflected that fatty acids, especially long chain unsaturated fatty acids, were critical for FFE-derived neuroprotective effects. Sphingolipids are essential for cellular membranes and sphingolipid disorder could induce central and the peripheral nervous systems degeneration in DEGS1 gene mutation [33]. Sphingolipids could also affect the steroid biosynthetic pathway at multiple levels [34]. Besides, unsaturated fatty acids like arachidonic acid and its metabolites were also decreased upon FFE treatment. Studies indicated that arachidonic acid could be disturbed in the brain after 6 days of LPS exposure [35], and the released arachidonic acid can be converted into pro-inflammatory lipid mediators, such as prostaglandin [36], showing that this increase is transient downregulated.

In conclusion, our results illustrated that FFE hold promising medicinal potential for protection of neuroinflammation through the inhibition of genes including IL-1ßand IL-6, as well as upregulation of metabolites like levodopadopamine, docosahexaenoic acid, gamma hydroxybutyric acid, 5-hydroxy-L-tryptophan, and alpha-tocopherol. Therefore, these preliminary results provided attractive molecular mechanism predictions for FFE in neuroinflammation protection of LPS-induced BV-2 cells. More experiments are needed for supporting and further exploring regulatory mechanism details and specific pathological processes.

Supplementary Materials: The following are available online at https:/ / www.mdpi.com/article/ 10.3390/app11178155/s1.

Author Contributions: X.Z. and J.Z. conceived and designed the study, X.Z. and X.W. collected, analyzed the data and wrote the paper, S.Z. cultured the cells and prepared metabolomics samples, X.L. (Xu Li), Z.L., X.L. (Xiaoxiao Liu), H.L. and X.C. collected and analyzed metabolomics data. All authors have read and agreed to the published version of the manuscript.

Funding: This research received no external funding.

Institutional Review Board Statement: The cell and animal experiment protocol was approved by the animal ethics committee of Xi'an Jiaotong University's School of Life Science and Technology (approval Nr. SCXK (Shaan) 2017-003).

Acknowledgments: This research work was supported by the Start-up fund from Northwest Normal University to Xinliang ZHU, Project for Enhancing the Research Capability of Young Teachers in Northwest Normal University to Xinliang ZHU (No. 5007/436) and Project for promoting the innovative capability of college and university from Gansu Province Education Department to Xinliang ZHU (No. 2060).

Conflicts of Interest: There is no conflict of interest.

\section{References}

1. Labzin, L.I.; Heneka, M.T.; Latz, E. Innate Immunity and Neurodegeneration. Annu. Rev. Med. 2018, 69, 437-449. [CrossRef]

2. Ransohoff, R.M. How neuroinflammation contributes to neurodegeneration. Science 2016, 353, 777-783. [CrossRef] [PubMed]

3. Lue, L.F.; Walker, D.G.; Brachova, L.; Beach, T.G.; Rogers, J.; Schmidt, A.M.; Stern, D.M.; Yan, S.D. Involvement of microglial receptor for advanced glycation endproducts (RAGE) in Alzheimer's disease: Identification of a cellular activation mechanism. Exp. Neurol. 2001, 171, 29-45. [CrossRef]

4. Gordon, R.; Albornoz, E.A.; Christie, D.C.; Langley, M.R.; Kumar, V.; Mantovani, S.; Robertson, A.A.B.; Butler, M.S.; Rowe, D.B.; O'Neill, L.A.; et al. Inflammasome inhibition prevents $\alpha$-synuclein pathology and dopaminergic neurodegeneration in mice. Sci. Transl. Med. 2018, 10, eaah4066. [CrossRef] 
5. Crotti, A.; Glass, C.K. The choreography of neuroinflammation in Huntington's disease. Trends Immunol. $2015,36,364-373$. [CrossRef] [PubMed]

6. Badshah, H.; Ikram, M.; Ali, W.; Ahmad, S.; Hahm, J.R.; Kim, M.O. Caffeine May Abrogate LPS-Induced Oxidative Stress and Neuroinflammation by Regulating Nrf2/TLR4 in Adult Mouse Brains. Biomolecules 2019, 9, 719. [CrossRef]

7. Yu, L.F.; Li, X.R.; Liu, S.Y.; Xu, G.W.; Liang, Y.Z. Comparative analysis of essential components between the herbal pair Radix Saposhnikoviae-Rhizoma seu Radix Notopterygii and its single herbs by GC-MS combined with a chemometric resolution method. Anal. Methods Adv. Methods Appl. 2009, 1, 45-51. [CrossRef]

8. Wang, S.; Hu, Y.; Tan, W.; Wu, X.; Chen, R.; Cao, J.; Chen, M.; Wang, Y. Compatibility art of traditional Chinese medicine: From the perspective of herb pairs. J. Ethnopharmacol. 2012, 143, 412-423. [CrossRef]

9. Dennis, G., Jr.; Sherman, B.T.; Hosack, D.A.; Yang, J.; Gao, W.; Lane, H.C.; Lempicki, R.A. DAVID: Database for Annotation, Visualization, and Integrated Discovery. Genome Biol. 2003, 4, P3. [CrossRef] [PubMed]

10. Jiang, X.W.; Liu, W.W.; Wu, Y.T.; Wu, Q.; Lu, H.Y.; Xu, Z.H.; Gao, H.Y.; Zhao, Q.C. Notopterygium incisum extract (NRE) rescues cognitive deficits in APP/PS1 Alzhneimer's disease mice by attenuating amyloid-beta, tau, and neuroinflammation pathology. $J$. Ethnopharmacol. 2020, 249, 112433. [CrossRef]

11. Chang, C.Z.; Wu, S.C.; Kwan, A.L.; Lin, C.L. 4'-O- $\beta$-D-glucosyl-5-O-methylvisamminol, an active ingredient of Saposhnikovia divaricata, attenuates high-mobility group box 1 and subarachnoid hemorrhage-induced vasospasm in a rat model. Behav. Brain Funct. 2015, 11, 28. [CrossRef]

12. Zheng, G.Q. Therapeutic history of Parkinson's disease in Chinese medical treatises. J. Altern. Complement. Med. 2009, 15, 1223-1230. [CrossRef] [PubMed]

13. Kim, H. Neuroprotective herbs for stroke therapy in traditional eastern medicine. Neurol. Res. 2005, 27, 287-301. [CrossRef]

14. Wang, H.; Wang, L.; Zhang, N.; Zhang, Q.; Zhao, H.; Zhang, Q. Houshiheisan compound prescription protects neurovascular units after cerebral ischemia. Neural Regen. Res. 2014, 9, 741-748. [CrossRef] [PubMed]

15. Kim, M.G.; Choi, J.H.; Lim, J.P.; Kim, D.K.; Shin, T.Y.; Boo, Y.; Kim, S.Y.; Kim, H.; Ha, E.; Park, H.K.; et al. Protective effects of a novel herbal decoction on focal cerebral ischemia in a rodent model. Neurol. Res. 2007, 29 (Suppl. S1), S16-S22. [CrossRef]

16. Sun, X.; Zhang, T.; Zhao, Y.; Cai, E.; Zhu, H.; Liu, S. Corrigendum to 'The protective effect of 5-O-methylvisammioside on LPSinduced depression in mice by inhibiting the over activation of BV-2 microglia through Nf-kB/IкB- $\alpha$ pathway' [Phytomedicine 79 (2020) 153348]. Phytomedicine 2021, 85, 153506. [CrossRef] [PubMed]

17. Meng, F.; Yu, W.; Duan, W.; Wang, T.; Liu, Y. Dexmedetomidine attenuates LPS-mediated BV2 microglia cells inflammation via inhibition of glycolysis. Fundam. Clin. Pharmacol. 2020, 34, 313-320. [CrossRef] [PubMed]

18. Lee, J.S.; Jeon, Y.J.; Kang, J.Y.; Lee, S.K.; Lee, H.D.; Son, C.G. Aquilariae Lignum Methylene Chloride Fraction Attenuates IL-1 $\beta$-Driven Neuroinflammation in BV2 Microglial Cells. Int. J. Mol. Sci. 2020, 21, 5465. [CrossRef]

19. Zhou, J.; Chen, J.; Hu, C.; Xie, Z.; Li, H.; Wei, S.; Wang, D.; Wen, C.; Xu, G. Exploration of the serum metabolite signature in patients with rheumatoid arthritis using gas chromatography-mass spectrometry. J. Pharm. Biomed. Anal. 2016, 127, 60-67. [CrossRef] [PubMed]

20. Priori, R.; Scrivo, R.; Brandt, J.; Valerio, M.; Casadei, L.; Valesini, G.; Manetti, C. Metabolomics in rheumatic diseases: The potential of an emerging methodology for improved patient diagnosis, prognosis, and treatment efficacy. Autoimmun. Rev. 2013, 12, 1022-1030. [CrossRef]

21. Li, R.; Guo, L.X.; Li, Y.; Chang, W.Q.; Liu, J.Q.; Liu, L.F.; Xin, G.Z. Dose-response characteristics of Clematis triterpenoid saponins and clematichinenoside AR in rheumatoid arthritis rats by liquid chromatography/mass spectrometry-based serum and urine metabolomics. J. Pharm. Biomed. Anal. 2017, 136, 81-91. [CrossRef]

22. Levite, M. Neurotransmitters activate T-cells and elicit crucial functions via neurotransmitter receptors. Curr. Opin. Pharmacol. 2008, 8, 460-471. [CrossRef]

23. Sarkar, C.; Basu, B.; Chakroborty, D.; Dasgupta, P.S.; Basu, S. The immunoregulatory role of dopamine: An update. Brain Behav. Immun. 2010, 24, 525-528. [CrossRef]

24. Zhang, Y.; Jiang, X.; Qin, C.; Cuevas, S.; Jose, P.A.; Armo, I. Dopamine D2 receptors' effects on renal inflammation are mediated by regulation of PP2A function. American journal of physiology. Renal Physiol. 2016, 310, F128-F134. [CrossRef] [PubMed]

25. Orr, S.K.; Palumbo, S.; Bosetti, F.; Mount, H.T.; Kang, J.X.; Greenwood, C.E.; Ma, D.W.; Serhan, C.N.; Bazinet, R.P. Unesterified docosahexaenoic acid is protective in neuroinflammation. J. Neurochem. 2013, 127, 378-393. [CrossRef]

26. Schober, M.E.; Requena, D.F.; Casper, T.C.; Velhorst, A.K.; Lolofie, A.; McFarlane, K.E.; Otto, T.E.; Terry, C.; Gensel, J.C. Docosahexaenoic acid decreased neuroinflammation in rat pups after controlled cortical impact. Exp. Neurol. 2019, 320, 112971. [CrossRef]

27. Wong, C.G.T.; Chan, K.F.Y.; Gibson, K.M.; Snead, O.C. $\gamma$-Hydroxybutyric Acid. Toxicol. Rev. 2004, 23, 3-20. [CrossRef]

28. Schirinzi, T.; Martella, G.; Imbriani, P.; Di Lazzaro, G.; Franco, D.; Colona, V.L.; Alwardat, M.; Sinibaldi Salimei, P.; Mercuri, N.B.; Pierantozzi, M. Dietary Vitamin E as a protective factor for Parkinson's disease: Clinical and experimental evidence. Front. Neurol. 2019, 10, 148. [CrossRef]

29. Capuron, L.; Schroecksnadel, S.; Féart, C.; Aubert, A.; Higueret, D.; Barberger-Gateau, P.; Layé, S.; Fuchs, D. Chronic low-grade inflammation in elderly persons is associated with altered tryptophan and tyrosine metabolism: Role in neuropsychiatric symptoms. Biol. Psychiatry 2011, 70, 175-182. [CrossRef] [PubMed] 
30. Wang, X.; Sun, G.; Feng, T.; Zhang, J.; Huang, X.; Wang, T.; Xie, Z.; Chu, X.; Yang, J.; Wang, H. Sodium oligomannate therapeutically remodels gut microbiota and suppresses gut bacterial amino acids-shaped neuroinflammation to inhibit Alzheimer's disease progression. Cell Res. 2019, 29, 787-803. [CrossRef]

31. Obeid, R.; McCaddon, A.; Herrmann, W. The role of hyperhomocysteinemia and B-vitamin deficiency in neurological and psychiatric diseases. Clin. Chem. Lab. Med. 2007, 45, 1590-1606. [CrossRef] [PubMed]

32. Annibal, A.; Tharyan, R.G.; Schonewolff, M.F.; Tam, H.; Latza, C.; Auler, M.M.K.; Antebi, A. Regulation of the one carbon folate cycle as a shared metabolic signature of longevity. Nat. Commun. 2021, 12, 3486. [CrossRef] [PubMed]

33. Karsai, G.; Kraft, F.; Haag, N.; Korenke, G.C.; Hänisch, B.; Othman, A.; Suriyanarayanan, S.; Steiner, R.; Knopp, C.; Mull, M. DEGS1-associated aberrant sphingolipid metabolism impairs nervous system function in humans. J. Clin. Investig. 2019, 129, 1229-1239. [CrossRef]

34. Lucki, N.C.; Sewer, M.B. Multiple roles for sphingolipids in steroid hormone biosynthesis. Lipids Health Dis. 2008, 49, 387-412.

35. Lee, H.; Villacreses, N.E.; Rapoport, S.I.; Rosenberger, T.A. In vivo imaging detects a transient increase in brain arachidonic acid metabolism: A potential marker of neuroinflammation. J. Neurochem. 2004, 91, 936-945. [CrossRef]

36. Jarc, E.; Petan, T. A twist of FATe: Lipid droplets and inflammatory lipid mediators. Biochimie 2020, 169, 69-87. [CrossRef] 